\title{
SIMILARITY SOLUTION FOR COMBINED FREE-FORCED CONVECTION PAST A VERTICAL POROUS PLATE IN A POROUS MEDIUM WITH A CONVECTIVE SURFACE BOUNDARY CONDITION
}

\author{
P. GARG \\ Department of Mathematics, KM College, University of Delhi \\ Delhi-110007, INDIA \\ G.N. PUROHIT \\ Department of Mathematics, Banasthali Vidyapith \\ Banasthali, Rajasthan-304022, INDIA \\ R.C. CHAUDHARY* \\ Department of Mathematics, University of Rajasthan \\ Jaipur, Rajasthan-302004, INDIA \\ E-mail: rcchaudhary@rediffmail.com
}

\begin{abstract}
This paper studies the mathematical implications of the two dimensional viscous steady laminar combined free-forced convective flow of an incompressible fluid over a semi infinite fixed vertical porous plate embedded in a porous medium. It is assumed that the left surface of the plate is heated by convection from a hot fluid which is at a temperature higher than the temperature of the fluid on the right surface of the vertical plate. To achieve numerical consistency for the problem under consideration, the governing non linear partial differential equations are first transformed into a system of ordinary differential equations using a similarity variable and then solved numerically under conditions admitting similarity solutions. The effects of the physical parameters of both the incompressible fluid and the vertical plate on the dimensionless velocity and temperature profiles are studied and analysed and the results are depicted both graphically and in a tabular form. Finally, algebraic expressions and the numerical values are obtained for the local skin- friction coefficient and the local Nusselt number.
\end{abstract}

Key words: combined convection, fixed vertical porous plate, skin-friction coefficient, Nusselt number, convective surface boundary condition, porous medium.

\section{Introduction}

In mixed convection flow situations both forced and free convection effects are of comparable order. It occurs in many transport processes and continues to be a topic of huge interest for researchers and scientists due to its wide application in several industrial and technical fields. This flow plays an important role, for example, in solar central receivers exposed to winds, nuclear reactors cooled during emergency shutdown, electronic devices cooled by fans and heat exchangers placed in a low velocity environment, manufacturing processes, process industries etc. In addition to these, the mixed convection flow exists in atmosphere when there is heat transfer from bodies moving through the atmosphere and also in oceanic circulation. Such flows transpire when the effect of buoyancy forces, in forced convection flow, becomes significant due to a large temperature difference between the wall and the ambient fluid.

An extensive study related to a free, forced and mixed convection boundary layer flow in a porous and non-porous medium can be found in literature [1-4]. A simple model of a mixed convective flow is the two-dimensional boundary layer flow over a flat plate in a uniform free stream. Some of the early studies of such flow can be found in papers [5-8]. Ali and Yousef [9] considered the problem of laminar mixed

\footnotetext{
* To whom correspondence should be addressed
} 
convection from a continuously moving vertical plate with suction/injection. Similar flow situations were studied for micropolar fluids by Gorla et al. $[10,11]$ and for surface embedded in porous medium by Alam et al. [12]. In all these papers, the velocity and thermal boundary layer for the case of constant surface temperature or variable temperature and heat flux at the surface have been considered.

Recently many papers relating to the convective boundary condition at the plate have been published. Aziz was the first to attempt a similarity solution for a laminar boundary layer flow over a flat plate with a convective surface boundary condition and demonstrated through his paper [14] that a similarity solution is possible if the convective heat transfer associated with the hot fluid on the lower surface of the plate is proportional to $x^{-1 / 2}$. Makinde [15] considered the problem of a free convection flow and heat transfer from a moving vertical plate with internal heat generation and a convective boundary condition. Ibrahim and Reddy [16] extended the problem by Makinde further to explore the effects of thermal radiation, viscous dissipation and chemical reaction on the similarity solutions for the same flow problem. Fazlina and Ishak [17] examined the problem of a mixed convection boundary layer flow for a vertical plate with a convective boundary condition.

The present paper considers the problem of the combined free-forced convection boundary layer flow past a porous vertical plate embedded in a porous medium and with a convective thermal boundary condition. The governing non-linear partial differential equations are first transformed into a system of ordinary differential equations with the help of a similarity variable and then solved numerically. The effects of the local Biot number, Grashof number, Prandtl number, permeability parameter and suction parameter on the velocity and temperature profiles are studied and illustrated through graphs. Algebraic expressions and numerical values are obtained for the local skin-friction coefficient and local Nusselt number along the surface of the plate.

\section{Mathematical analysis}

Consider a two dimensional viscous steady laminar mixed convective flow of an incompressible fluid over a semi infinite fixed vertical porous plate placed in a porous medium. The left surface of the plate is heated by convection from a hot fluid which is at a higher temperature $T_{f}$ while the right surface of the plate is in contact with a cold fluid at a temperature $T_{\infty}$, as shown in Fig.1. Let the hot fluid on the left surface of the plate provide a heat transfer coefficient of $h_{f}$. It is assumed that the flow is vertically upwards along the vertical plate which is taken along the $x$-axis while the $y$-axis is assumed to be normal to the vertical plate. It is also assumed that the free stream velocity $U_{\infty}$, parallel to the vertical plate, is constant.

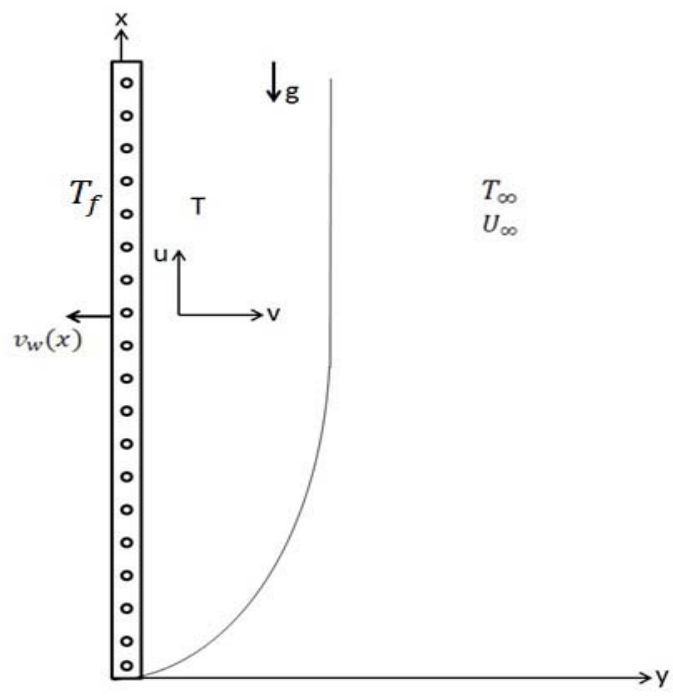

Fig.1. 
Then, under the Boussinesq approximations for fluid density variations and the boundary layer conditions, the equations of continuity, momentum and energy relating the flow are

$$
\begin{aligned}
& \frac{\partial u}{\partial x}+\frac{\partial v}{\partial y}=0, \\
& u \frac{\partial u}{\partial x}+v \frac{\partial u}{\partial y}=v \frac{\partial^{2} u}{\partial y^{2}}+g \beta\left(T-T_{\infty}\right)-\frac{v}{K^{\prime}} u, \\
& u \frac{\partial T}{\partial x}+v \frac{\partial T}{\partial y}=\alpha \frac{\partial^{2} T}{\partial y^{2}}
\end{aligned}
$$

where $u$ and $v$ are the $x$ (along the plate) and the $y$ (normal to the plate) components of the velocities, respectively, $T$ is the temperature, $\mathrm{u}$ - the kinematic viscosity of the fluid, $\rho$ - the fluid density, $\alpha\left(=\frac{k}{\rho c_{p}}\right)$ the thermal diffusivity of the fluid, $k$ - the thermal conductivity, $\beta$ - the thermal expansion coefficient and $K^{\prime}$ is the permeability of the porous medium.

The boundary conditions at the plate surface and far into the cold fluid can be expressed as

$$
\begin{aligned}
& u=0, \quad v=v_{w}(x), \quad-k \frac{\partial T}{\partial y}=h_{f}\left(T_{f}-T(x, 0)\right) \quad \text { at } \quad y=0, \\
& u \rightarrow U_{\infty}, \quad T \rightarrow T_{\infty}, \quad \text { as } \quad y \rightarrow \infty .
\end{aligned}
$$

In order to obtain the similarity solution of the problem, we define an independent variable $\eta$ and dependent functions $f(\eta)$ and $\theta(\eta)$ as

$$
\eta=y\left(\frac{U_{\infty}}{v x}\right)^{1 / 2}, \quad f(\eta)=\frac{\psi}{\sqrt{U_{\infty} v x}}, \quad \theta=\frac{T-T_{\infty}}{T_{f}-T_{\infty}}
$$

where $\psi$ is the stream function defined by $u=\frac{\partial \psi}{\partial y}$ and $v=-\frac{\partial \psi}{\partial x}$ which identically satisfies Eq.(2.1).

Equations $(2.1)-(2.5)$ reduce to

$$
\begin{aligned}
& f^{\prime \prime \prime}+\frac{1}{2} f f^{\prime \prime}+G_{s} \theta-K f^{\prime}=0, \\
& \theta^{\prime \prime}+\frac{1}{2} \operatorname{Pr} f \theta^{\prime}=0, \\
& f(0)=f_{w}, \quad f^{\prime}(0)=0, \quad \theta^{\prime}(0)=-B_{i}[1-\theta(0)], \quad f^{\prime}(\infty)=1, \quad \theta(\infty)=0
\end{aligned}
$$


where

$$
\begin{aligned}
& f_{w}=-2 v_{w}(x)\left(\frac{x}{v U_{\infty}}\right)^{1 / 2} \quad \text { is the suction parameter, } \\
& \mathrm{Bi}=\frac{h_{f}}{k}\left(\frac{v x}{U_{\infty}}\right)^{1 / 2} \quad \text { is the local Biot number, } \\
& G s=\frac{g \beta\left(T_{f}-T_{\infty}\right) x}{U_{\infty}^{2}}=\frac{\mathrm{Gr}}{\mathrm{Re}^{2}} \quad \text { is the temperature buoyancy parameter, } \\
& \mathrm{Gr}=\frac{g \beta\left(T_{f}-T_{\infty}\right) x^{3}}{v^{2}} \quad \text { is the local Grashof number, } \\
& \operatorname{Re}=\frac{U_{\infty} x}{v} \quad \text { is the local Reynolds number, } \\
& K=\frac{U x}{K^{\prime} U_{\infty}} \quad \text { is the local permeability parameter, } \\
& \operatorname{Pr}=\frac{v}{\alpha} \quad \text { is the Prandtl number. }
\end{aligned}
$$

In order to have a similarity solution, the parameters $G s, K, f_{w}, B_{i}$ must be constant. We thus assume that $\beta$ is proportional to $x^{-1}, K^{\prime}$ is proportional to $x$, the suction velocity $v_{w}$ and the heat transfer coefficient $h_{f}$ are proportional to $x^{-1 / 2}$.

We therefore take

$$
\beta=a x^{-1}, \quad K^{\prime}=b x, \quad v_{w}=c x^{-1 / 2}, \quad h_{f}=d x^{-1 / 2}
$$

where $a, b, c, d$ are constants with appropriate dimensions.

With these assumptions, we have

$$
\mathrm{Gs}=\frac{a g\left(T_{f}-T_{\infty}\right)}{U_{\infty}{ }^{2}}, \quad K=\frac{v}{b U_{\infty}}, \quad f_{w}=-2 c\left(\frac{1}{v U_{\infty}}\right)^{1 / 2}, \quad \mathrm{Bi}=\frac{d}{k}\left(\frac{v}{U_{\infty}}\right)^{1 / 2} .
$$

\section{The skin- friction coefficient and Nusselt number}

The local skin-friction coefficient and local Nusselt-number indicate physically the wall shear stress and the rate of heat transfer, respectively.

The wall skin- friction is: $\tau_{w}=\mu\left(\frac{\partial u}{\partial y}\right)_{y=0}=\mu U_{\infty} \sqrt{\frac{U_{\infty}}{v x}} f^{\prime \prime}(0)$. 
The local skin-friction coefficient is: $C_{f}=\frac{2}{\rho U_{\infty}^{2}} \tau_{w}=2(\mathrm{Re})^{-1 / 2} f^{\prime \prime}(0)$.

The heat flux is: $\quad q_{w}=-k\left(\frac{\partial T}{\partial y}\right)_{y=0}=-k \Delta T \sqrt{\frac{U_{\infty}}{v x}} \theta^{\prime}(0)$.

The local Nusselt number is: $\mathrm{Nu}=\frac{x}{k \Delta T} q_{w}=-\operatorname{Re}^{1 / 2} \theta^{\prime}(0)$

where $\Delta T=T_{f}-T_{\infty}$.

\section{Results and discussion}

The system of coupled non linear boundary value problem Eqs (2.7)-(2.8) together with the boundary conditions (2.9) has been solved numerically. Numerical calculations have been carried out for different values of the parameters $\mathrm{Gs}, \mathrm{Bi}, f w, K$ and Pr. From the numerical computations the values of the local skin friction, local Nusselt number which are proportional to $f^{\prime \prime}(0),-\theta^{\prime}(0)$ and plate surface temperature $\theta(0)$ are calculated and listed in Tabs $1-5$.The behaviour of these due to various parameters is self evident from these tables. Physically, a positive sign of the skin friction coefficient implies that the force exerts a drag force on the right surface of the plate.

The dimensionless parameter $G s$ represents the free, forced and combined (free- forced) convection regimes. For pure forced convection $G s \ll 1$, for combined free- forced convection $G s=1$ and for pure free convection $G s \gg 1$. The Prandtl number is a measure of the ratio of diffusivities while the Biot number is a measure of heat losses to the surroundings. As Bi becomes smaller, there is more heat available. The positive values of the parameters are used in our computations.

Computations showing variation of $f^{\prime \prime}(0),-\theta^{\prime}(0)$ and $\theta(0)$ at the plates for different parameter values:

Table 1. Effect of Biot number for $\operatorname{Pr}=0.71, G s=1.0, f w=0.5, K=0.05$.

\begin{tabular}{|l|l|l|l|}
\hline \multicolumn{1}{|c|}{$\mathrm{Bi}$} & \multicolumn{1}{c|}{$f^{\prime \prime}(0)$} & \multicolumn{1}{c|}{$-\theta^{\prime}(0)$} & \multicolumn{1}{c|}{$\theta(0)$} \\
\hline 0.1 & 0.620348 & 0.0811761 & 0.188239 \\
\hline 0.5 & 0.96951 & 0.24099 & 0.518019 \\
\hline 1.0 & 1.12166 & 0.323452 & 0.676548 \\
\hline 5.0 & 1.33339 & 0.45007 & 0.909986 \\
\hline
\end{tabular}

Table 2. Effect of Prandtl Number for $G s=1.0, \mathrm{Bi}=1.0, f w=0.5, K=0.05$.

\begin{tabular}{|l|l|l|l|}
\hline \multicolumn{1}{|c|}{$\operatorname{Pr}$} & \multicolumn{1}{c|}{$f^{\prime \prime}(0)$} & \multicolumn{1}{c|}{$-\theta^{\prime}(0)$} & \multicolumn{1}{c|}{$\theta(0)$} \\
\hline 0.1 & 1.75758 & 0.168256 & 0.831744 \\
\hline 0.71 & 1.12166 & 0.323452 & 0.676548 \\
\hline 3.0 & 0.706698 & 0.515232 & 0.484768 \\
\hline 10.0 & 0.474388 & 0.730034 & 0.269966 \\
\hline
\end{tabular}


Table 3. Effect of $\mathrm{Gs}$ for $\operatorname{Pr}=0.71, \mathrm{Bi}=1.0, f w=0.5, K=0.05$.

\begin{tabular}{|l|l|l|l|}
\hline \multicolumn{1}{|c|}{$G s$} & \multicolumn{1}{|c|}{$f^{\prime \prime}(0)$} & \multicolumn{1}{c|}{$-\theta^{\prime}(0)$} & \multicolumn{1}{c|}{$\theta(0)$} \\
\hline 0.1 & 0.365528 & 0.235322 & 0.764678 \\
\hline 0.5 & 0.719813 & 0.261349 & 0.738651 \\
\hline 1.0 & 1.07266 & 0.279963 & 0.720037 \\
\hline 2.0 & 1.65666 & 0.302923 & 0.697077 \\
\hline
\end{tabular}

Table 4. Effect of the suction parameter for $\operatorname{Pr}=0.71, G s=1.0, \mathrm{Bi}=1.0, K=0.05$.

\begin{tabular}{|l|l|l|l|}
\hline \multicolumn{1}{|c|}{$f_{w}$} & \multicolumn{1}{|c|}{$f^{\prime \prime}(0)$} & \multicolumn{1}{c|}{$-\theta^{\prime}(0)$} & \multicolumn{1}{c|}{$\theta(0)$} \\
\hline 0.0 & 1.06128 & 0.269068 & 0.730932 \\
\hline 0.5 & 1.12166 & 0.323452 & 0.676548 \\
\hline 1.0 & 1.19649 & 0.376247 & 0.623753 \\
\hline 1.5 & 1.29278 & 0.425604 & 0.574396 \\
\hline
\end{tabular}

Table 5. Effect of permeability parameter for $\operatorname{Pr}=0.71, G s=1.0, \mathrm{Bi}=1.0, f w=0.5$.

\begin{tabular}{|l|l|l|l|}
\hline \multicolumn{1}{|c|}{$K$} & \multicolumn{1}{c|}{$f^{\prime \prime}(0)$} & \multicolumn{1}{c|}{$-\theta^{\prime}(0)$} & \multicolumn{1}{c|}{$\theta(0)$} \\
\hline 0.0 & 0.907784 & 0.319461 & 0.680539 \\
\hline 0.05 & 0.793189 & 0.309847 & 0.690153 \\
\hline 0.1 & 0.696587 & 0.30026 & 0.696587 \\
\hline 0.5 & 0.395412 & 0.248289 & 0.395412 \\
\hline
\end{tabular}

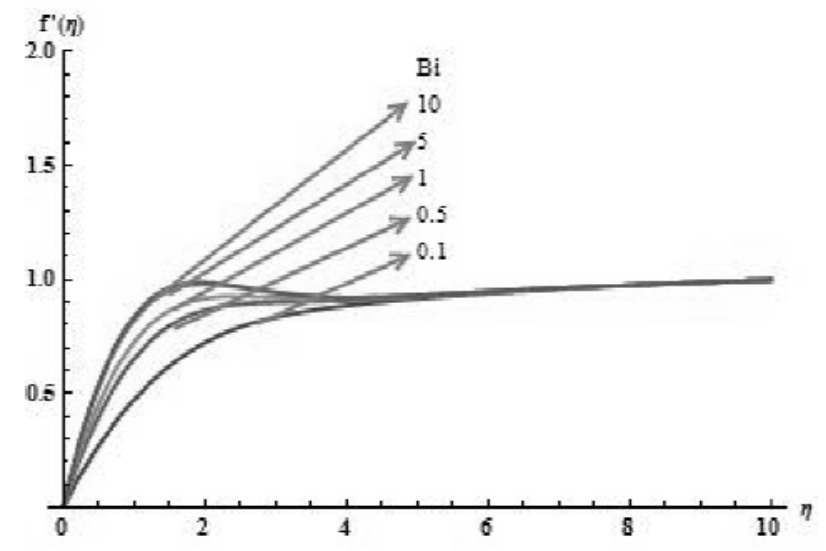

Fig.1a. Variation of the velocity component $f^{\prime}$ with Bi for $\operatorname{Pr}=0.71, f_{w}=1.5, G s=1.0, K=0.05$

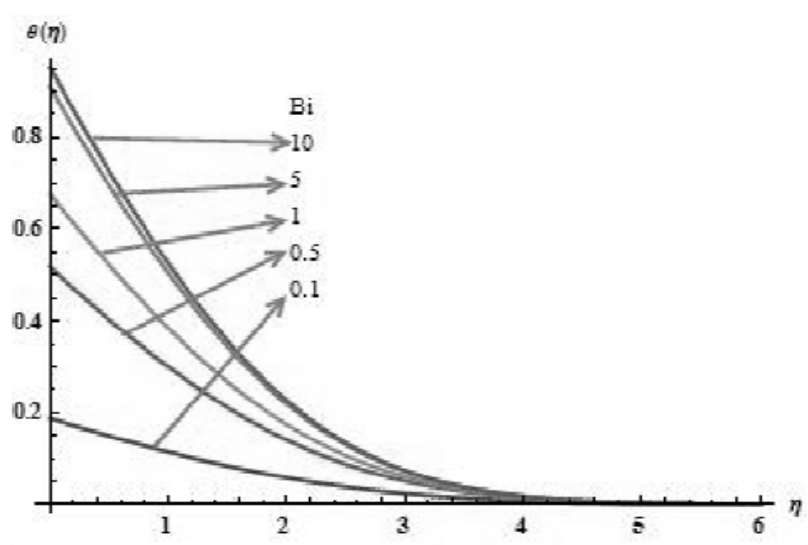

Fig.2a. Variation of the temperature $\theta$ with Bi for $\operatorname{Pr}=0.71, f_{w}=1.5, G s=1.0, K=0.05$. 


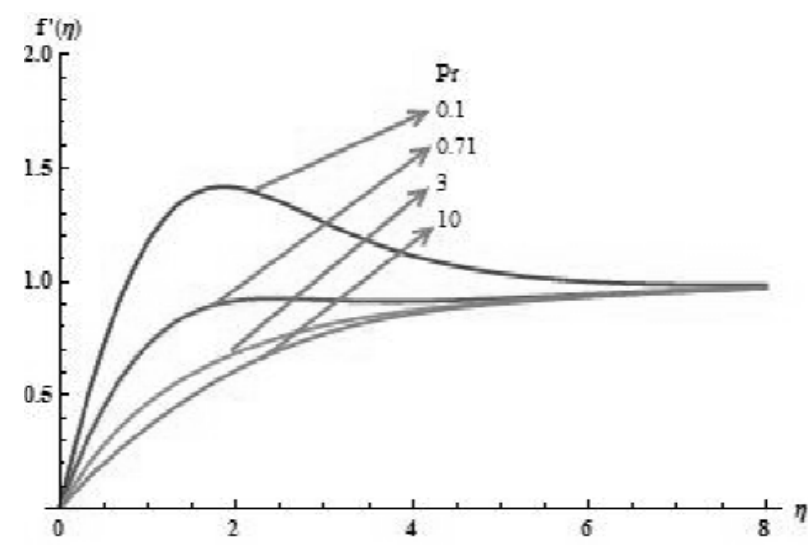

Fig.1b. Variation of the velocity component $f^{\prime}$ with $\operatorname{Pr} f_{w}=0.5, \mathrm{Bi}=1.0, G s=1.0, K=0.05$.

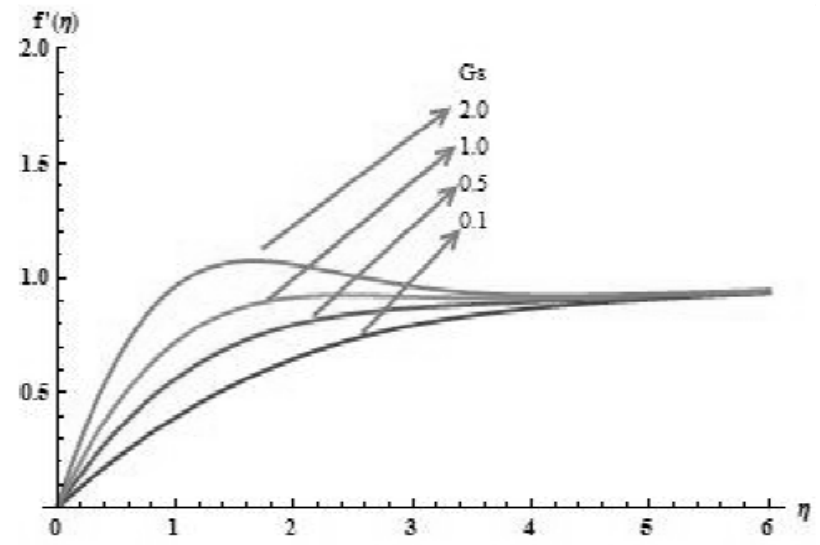

Fig.1c. Variation of the velocity component $f^{\prime}$ with $G s$ for $\operatorname{Pr}=0.71, \mathrm{Bi}=1.0, f_{w}=0.5, K=0.05$.

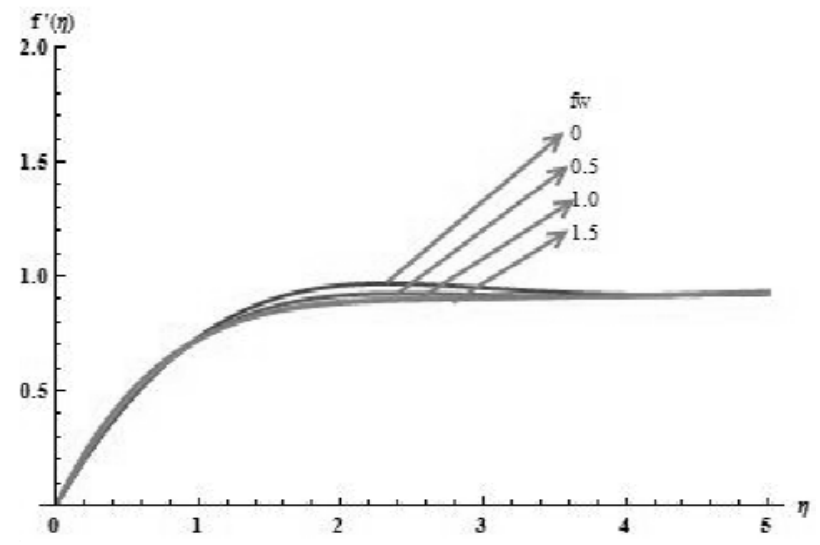

Fig.1d. Variation of the velocity component $f^{\prime}$ with $f_{w}$ for $\operatorname{Pr}=0.71, \mathrm{Bi}=1.0, G s=1.0, K=0.05$.

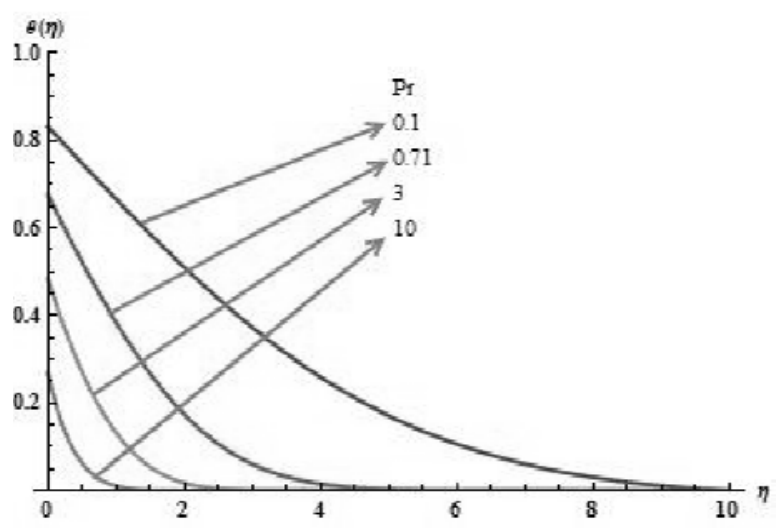

Fig.2b. Variation of the temperature $\theta$ with $\operatorname{Pr}$ for for $f_{w}=0.5, \mathrm{Bi}=1.0, G s=1.0, K=0.05$.

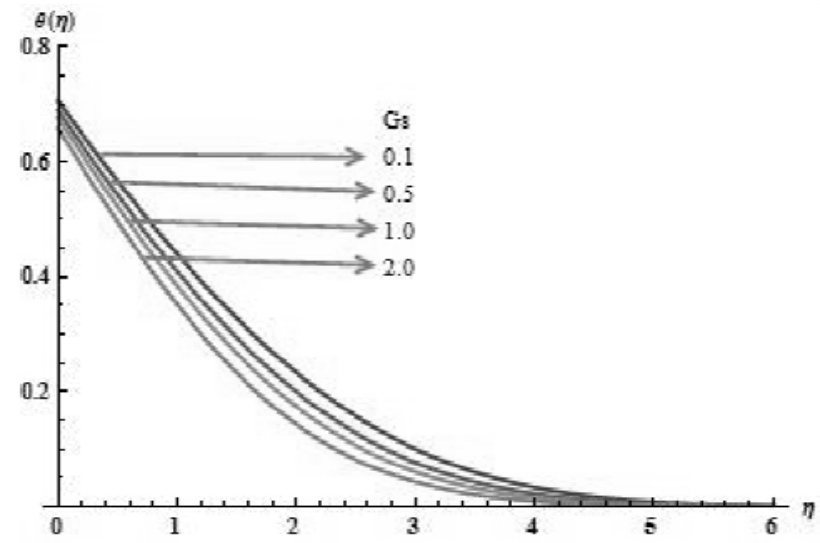

Fig.2c. Variation of the temperature $\theta$ with Gs with $G s$ for $\operatorname{Pr}=0.71, \mathrm{Bi}=1.0, f_{w}=0.5, K=0.05$.

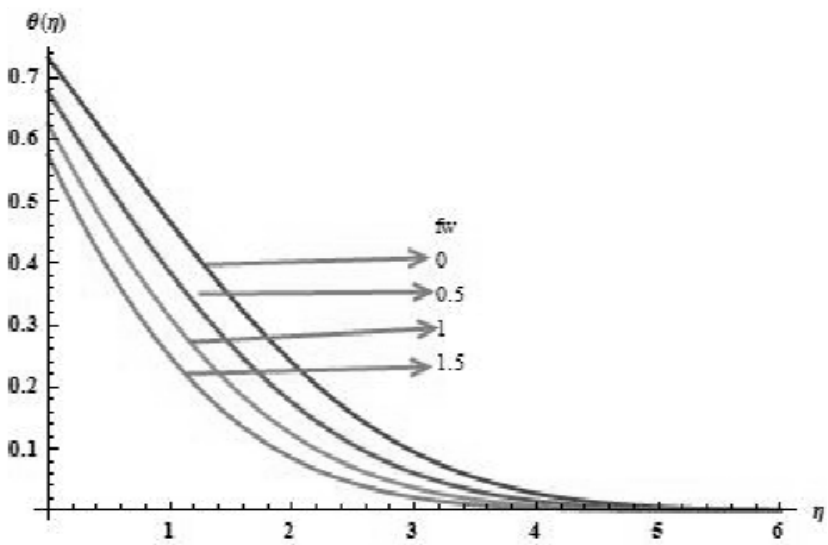

Fig.2d. Variation of the temperature $\theta$ with $f_{w}$ for $\operatorname{Pr}=0.71, \mathrm{Bi}=1.0, G s=1.0, K=0.05$. 


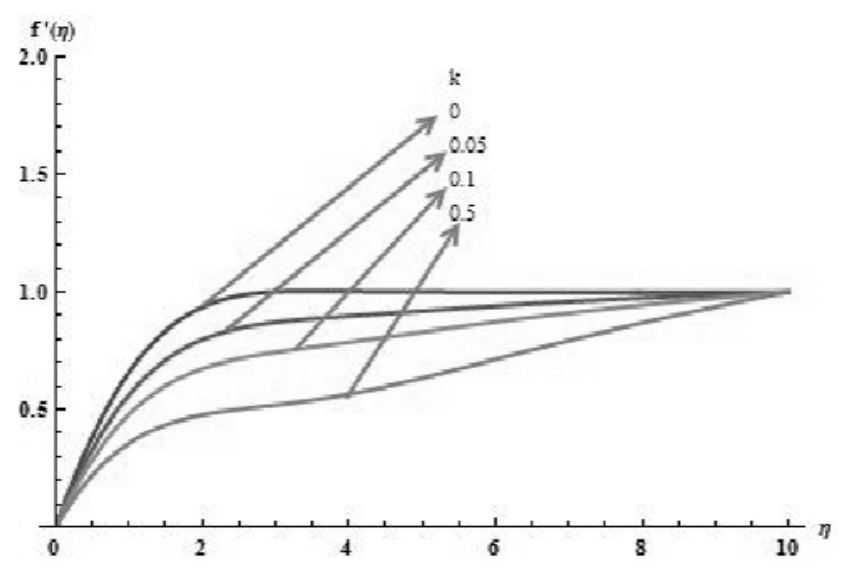

Fig.1e. Variation of the velocity component $f^{\prime}$ with $K$ for $\operatorname{Pr}=0.71, f_{w}=0.5, G s=1.0, \mathrm{Bi}=1.0$.

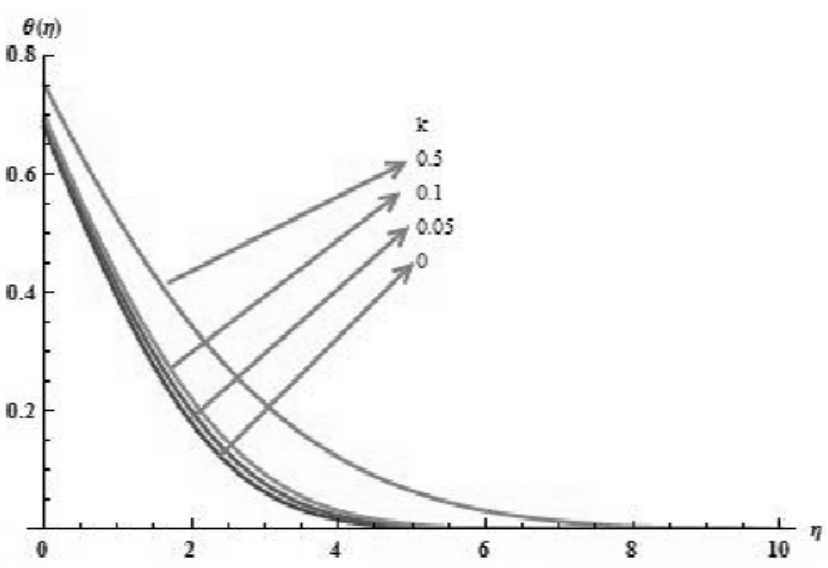

Fig.2e. Variation of the temperature $\theta$ with $K$ for $\operatorname{Pr}=0.71, f_{w}=0.5, G s=1.0, \mathrm{Bi}=1.0$.

The effects of various parameters on the velocity field and temperature field are well illustrated in the graphs plotted in Figs 1a-1e and 2a-2e respectively.

It can be seen from the Figs 1a-1e that the velocity profiles increase monotonically with the increase in the Biot number and the temperature buoyancy parameter. However, they decrease with the increase in the Prandtl number, permeability parameter and the suction parameter. The decrease of the velocity profile with the increase of the suction parameter indicates the usual fact that suction stabilizes the boundary layer growth.

Figures $2 \mathrm{a}-2 \mathrm{e}$ clearly show the effects of parameters on the temperature profiles. It can be seen that with the increase in the Biot number or the permeability parameter, the temperature profile increases but with the increase in the temperature buoyancy parameter, the Prandtl number or the suction parameter, the temperature profile decreases.

The decrease with the increase of the suction parameter indicates that sucking decelerated fluid particles through the porous wall reduces the growth of the fluid boundary layer as well as thermal boundary layers.

\section{Conclusions}

In this paper we have studied the two dimensional viscous steady laminar combined free-forced or mixed convective flow of an incompressible fluid across a semi infinite fixed vertical porous plate embedded in a porous medium assuming that the left surface of the plate is heated by convection from a hot fluid which is at a temperature higher than the temperature of the fluid present at the right surface of the vertical plate.

We observed that a similarity solution of the above problem is possible only if the convective heat transfer coefficient of the fluid heating the left surface of the plate and the suction velocity are both proportional to $x^{-1 / 2}$, the thermal expansion coefficient $\beta$ is proportional to $x^{-1}$ and the permeability of the porous medium is proportional to $x$.

Numerical solutions of the resulting similarity equations can be used to calculate the surface heat flux and skin friction coefficient by using the derived algebraic expressions. Also, as expected the surface temperature $\theta(\theta)$ increases as $\mathrm{Bi}$ increases as indeed is the case in Tab.1 and Fig.2a. This is because as $\mathrm{Bi}$ increases, the hot fluid side convection resistance decreases and consequently, the surface temperature $\theta(0)$ should increase. 


\section{Nomenclature}

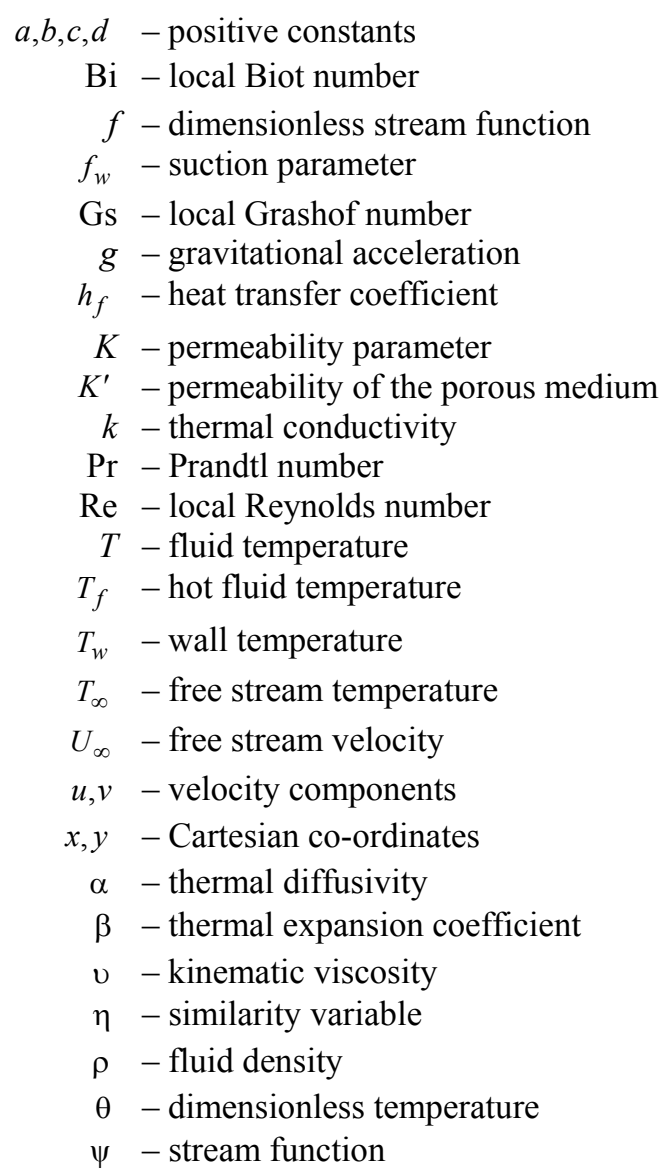

\section{References}

[1] Schlichting H. and Gersten K. (2000): Boundary Layer Theory. $-8^{\text {th }}$ Edition, Springer, Verlag Berlin Heidelberg.

[2] Nield D.A. and Bejan A. (2013): Convection in Porous Media. $-4^{\text {th }}$ Edition, New York: Springer.

[3] Vafai K. (2015): Handbook of Porous Media. - 3rd edition, CRC Press.

[4] Bird R.B., Stewart W.E. and Lightfoot E.N. (2007): Transport Phenomena. - Revised $2^{\text {nd }}$ Edition, John Wiley and Sons.

[5] Lloyd J.R. and Sparrow E.M. (1970): Combined forced and free convection flow on vertical surfaces. - Int. J. Heat Mass Transfer, vol.13, No.2, pp.434-438.

[6] Wilks G. (1973): Combined forced and free convection flow on vertical surfaces. - Int. J. Heat Mass Transfer, vol.16, No.10, pp.1958-1964.

[7] Raju M.S., Liu X.R. and Law C.K. (1984): A formulation of combined forced and free convection past horizontal and vertical surfaces. - Int. J. Heat Mass Transfer, vol.27, No.12, pp.2215-2224.

[8] Hossain M.A. and Ahmed M.U. (1990): MHD forced and free convection boundary layer flow near the leading edge. - Int. J. Heat Mass Transfer, vol.33, No.3, pp.571-575.

[9] Ali M. and Al-Yousef F. (1998): Laminar mixed convection from a continuously moving vertical surface with suction or injection. - Heat and Mass Transfer, vol.33, pp.301-306.

[10] Gorla R.S.R., Lin P.P. and Yang A.J. (1990): Asymptotic boundary layer solutions for mixed convection from a vertical surface in a micropolar fluid. - Int. J. Eng. Sci., vol.28, No.6, pp.525-533. 
[11] Gorla R.S.R. (1992): Mixed convection in a micropolar fluid along a vertical surface with uniform heat flux. - Int. J. Heat and Mass Transfer, vol.28, No.6, pp.525-533.

[12] Alam M.S., Rahman M.M. and Samad M.A. (2006): Numerical study of the combined free forced convection and mass transfer flow past a vertical porous plate in a porous medium with heat generation and thermal diffusion. - NonLinear Analysis: Modelling and Control, vol.11, No.4, pp.331-343.

[13] Bhattacharyya K., Mukhopadhya S. and Layek G.C. (2013): Similarity solution of mixed convective boundary layer, slip flow over a vertical plate. - Ain Shams Engineering Journal, vol.4, pp.299-305.

[14] Aziz A. (2009): A similarity solution for Laminar thermal boundary layer over a flat plate with a convective surface boundary condition. - Commun. Nonlinear Sci. Numer. Simulate, vol.14, pp.1064-1068.

[15] Makinde O.D. (2011): Similarity solution for natural convection from a moving vertical plate with internal heat generation and a convective boundary condition. - Thermal Science, vol.15, No.1, pp.S137-S143.

[16] Ibrahim S.M and Reddy N.B. (2013): Similarity solution of heat and mass transfer for natural convection over a moving vertical plate with internal heat generation and a convective boundary condition in the presence of thermal radiation, viscous dissipation, and chemical reaction. - Hindawi Publishing Corporation ISRN Thermodynamics, Article ID 790604, 10 pages, http://dx.doi.org/10.1155/2013/790604.

[17] Fazlina Aman and Anuar Ishak (2012): Mixed convection boundary layer flow towards a vertical plate with a convective surface boundary condition. - Hindawi Publishing Corporation Mathematical Problems in Engineering, Article ID 453457, 11 pages doi:10.1155/2012/453457.

Received: November 24, 2015

Revised: May 28, 2016 